\title{
Leptin Levels and its Relationship to Liver Dysfunctional Diseases and Hepatocellular Carcinoma
}

\author{
Ana Lúcia Prudêncio del Castilo ${ }^{1}$, Kelly Cristina Oliveira Jordão², Ayeska Espeschit Maia², \\ Rafael Perseghini Del Sarto ${ }^{3}$, Aline Maria Araújo Martins ${ }^{4,5 *}$ \\ ${ }^{1}$ University Hospital of Brasilia, HUB - University of Brasilia UNB - DF, Brazil \\ ${ }^{2}$ University of Brasilia $U N B-D F$, Brazil \\ ${ }^{3}$ Centro Universitário do Distrito Federal, Brasília UDF - DF, Brazil \\ ${ }^{4}$ Brazilian Center for Protein Research - LBQP, Molecular Pathology, Medicine College, University of Brasilia, UNB - DF, Brazil \\ ${ }^{5}$ Liver Transplantation Center, HUWC / Federal University of Ceara - UFC, Brazil
}

Received: September 01, 2016; Accepted: September 20, 2016; Published: September 26, 2016

*Corresponding author: Aline Maria Araujo Martins, Brazilian Center for Protein Research, Department of Cell Biology, University of Brasilia, UNB DF, Brazil; Tel: +55 6198186 9696; E-mail: alinemartins@ufc.br

\begin{abstract}
Obesity is an important risk factor for several types of cancer, including hepatocellular carcinoma. The amount of fat cells of an organism is proportional to the leptin levels, which transmit the information on stored energy, triggering the necessary control mechanisms; however, in obese individuals, a leptin resistance phenotype is observed. Obesity phenotype displays a proinflammatory response, enhancing the progression of liver insulin resistance in patients with steatohepatitis. Steatotic livers exhibit a dysfunctional lipidic stimulus, activating macrophages and Kupffer cells, producing growth factors, cytokines and chemokines, causing more proliferation and collagen synthesis, in a positive proinflammatory feedback. This lipidic imbalance alters leptin function, which plays an important role on TNF- $\alpha$ and IL- 6 regime, affecting Janus Kinase and STAT16 signal transducers; and phosphatidylinositol 3-kinase signaling pathway. These molecular deregulation progresses to liver fibrosis and cirrhosis especially due to upregulation of TGF- $\beta$ and collagen production, resulting in a cytoarchitecture breakdown, which compromises cellular function, increasing cirrhotic phenotype. The chronic momentum intensify local inflammatory responses, causing a compensation hepatocyte proliferation (injury proliferation), especially in obese individuals, facilitating the malignant transformation of affected hepatocytes and HCC development. In this context, this paper aims to review general information about the complexity machinery involved on this important tripod of lectin modulation, liver dysfunction due to lipidic imbalance and the progression of hepatocellular carcinoma.
\end{abstract}

Keywords: Leptin; Obesity; Liver Dysfuntional Diseases; NonAlcoholic Fatty Liver Disease; Steatosis; Hepatocellular Carcinoma

\section{Abbreviations}

AgRP- Argouti-Related Protein; AMPK - Adenosine Monophosphate-Activated Protein Kinase; CART - Cocaine and Anfetamine Regulated Transcript; HCC - Hepatocellular Carcinoma; IL-1 - Interleucin 1; IL-6 - Interleucin 6; kDa - kilo Daltons; JAK - Janus kinase; NASH - Non-Alcooholic
Steatohepatitis; NAFLD - Non-Alcooholic Fatty Liver Disease; NPY - Neuropeptide Y; PI3K - Phosphatidilinositol 3-Kinase; POMC - Pro-Opiomelanocortin; STAT - Signal Transducers And Activators Of Transcription; TG - Triglicerides; TNF- $\alpha$ - Tumor Necrosis Factor alpha ; TGF- $\beta$ - Transforming Growth Factor- $\beta$; $\alpha$-MSH - $\alpha$ - Melanoocyte Stimulating Hormone

\section{Introduction}

Obesity is an important risk factor for several types of cancer, including hepatocellular carcinoma (HCC) [1, 2-6]. Adipocytokines are the main body weight regulators; among them, leptin plays a central role since it is the product of the Ob gene unglycosylated protein $(16-\mathrm{kDa})$ and is part of the mechanisms of energy expenditure maintenance (food intake versus energy expenditure) [7]. Leptin is a multifunctional peptide hormone with neuroendocrine activity, insulin sensitivity, stress response, playing an important role in angiogenesis, bone formation and immune response modulation $[8,9,10]$.

The amount of fat cells of an organism is proportional to the leptin levels, which transmit the information on stored energy to the hypothalamus, triggering the necessary control mechanisms $[11,3]$. High leptin levels decrease appetite and increase energy expenditure and, on the other hand, low systemic leptin level increases appetite and decreases energy expenditure. However, in obese individuals, these mechanisms are compromised, because even with high serum leptin levels they resist its effects, which characterize the leptin resistance phenotype [12,13].

Regarding hepatic cancer, there are approximately 626,000 new cases of primary liver cancer worldwide, almost all represented by hepatocellular carcinoma (HCC). With a high mortality ratio (0.93), 598,000 of these patients die from this cancer each year, making HCC the third most common cause of cancer death [14]. The etiological factors associated with hepatocellular carcinoma are chronic viral infection with hepatitis 
B and C, chronic alcoholism, Non-Alcoholic Steatohepatitis (NASH) and contaminated food (e.g. aflatoxins) [15-18].

Morbidly obese patients have a prevalence of more than $90 \%$ of changes in liver histology. The histological prevalence is fatty liver, hepatic steatosis, which is the most mild of Non-Alcoholic Fatty Liver Disease (NAFLD) in individuals who do not consume alcohol or consume minimal amounts (20g ethanol/week) [1,9].

Abdominal obesity, dyslipidemia and high blood pressure are known as metabolic syndromes and are associated with a chronic proinflammatory condition $[19,20]$. The most important factors of metabolic syndrome are genetic factors and lifestyle, such as sedentary behavior, leading to subsequent fat accumulation, which could result in other diseases and comorbidities [15, 21].

The increase of fatty acids in hepatocytes that are secreted by adipose tissue can be a major cause of obesity induced metabolic syndrome [22]. The liver synthesizes more triglycerides, but cannot export them efficiently. As a result, triglycerides accumulate in the liver parenchymal cells (hepatocytes), leading to NASH-like phenotype, characterized by hepatic steatosis [23]. These triglycerides are associated with the endoplasmic reticulum (ER) stress on hepatic cells, which leads to hepatocyte lipoapoptosis [24].

Leptin has proven to be important in the progression of hepatic steatosis $[23,25,26]$. As a result of synergistic action of other adipocytokines such as tumor necrosis factor (TNF- $\alpha$ ), fatty liver may progress to steatohepatitis by lipoapoptosis induction, which leads to the recruitment of inflammatory cells, contributing to its progression and subsequently causing hepatocellular damage, such as cell swelling and appearance of Mallory bodies [27,25].

Leptin has an increasingly important role to play in a variety of human metabolic disorders and cancers. This is easily demonstrated by the elevated number of studies about this hormone and the emphasis on its importance in malignant events, such those found in published articles that has this descriptors ("leptin") related to the prevalence of hepatic steatosis in patients with hepatocellular carcinoma [11]. In this context, this paper aims to review general information about leptin, including its receptor and its related signaling pathways, by providing relevant information on the association between leptin and liver steatosis/steatohepatitis, as relevant factors associated with the establishment and progression of HCC.

\section{Leptin role and adipocity metabolic signals}

Cloning and sequencing performed in mice Ob gene and the discovery of leptin, in 1994 by the group of Dr. Friedman at Columbia University in New York, leveraged molecular knowledge in mammals' body fat control $[28,29]$.

Leptin activities due to its central receptors, especially on the hypothalamus, and on various other peripheral organs such as pancreas, liver, adipose tissue and immune system [11]. Leptin receptors are essential to the processes that lead to its performance, as they play critical roles in mediating pleiotropic effects on mammalian physiology. Untill nowadays, there are six splice variants of the leptin receptor gene: OB-R (a-e) and soluble short form (Ob-R - muB219) [30, 31]. These splice variants have different intracellular $\mathrm{C}$ termini, but identical extracellular leptin binding motifs.

Transcription and translation are performed in adipose tissue, placenta and gastrointestinal tract, and their product is directly related to the mass of adipose tissue. The circulating leptin levels in plasma are directly related to the amount of its mRNA in adipocytes) [30, 32].

The leptin debt is regulated by the adjustment of fat reserves, which act on the hypothalamus, specifically on the arcuate nucleus, provided with two populations of leptin sensitive neurons: i) appetite stimulants (orexigenic) secrete neuropeptide Y (NPY) and AgRP (Agouti related protein) and ii) appetite suppressants (anorexigenic) secrete CART (cocaine and amphetamine regulated transcript), and $\alpha-\mathrm{MSH}$ ( $\alpha$-Melanocyte Stimulating Hormone), derived from POMC (proopiomelanocortin) [33]. The binding of leptin decreases food intake and increases energy expenditure by stimulating the production of $\alpha$-MSH and CART (anorexigenic peptides) and inhibiting the synthesis of NPY and AgRP (orexigenic peptides) [8]. The opposite occurs when there is inappropriate fat reserve. The secretion of leptin increases food intake and the orexigenic neurons are no longer inhibited. In individuals with stable weight, the activities of these pathways are balanced [11].

Most obese individuals have high leptin levels, which contributes to the resistance to its effects. Studies about the relation between cerebrospinal-fluid (CSF) and serum leptin in human obesity suggest that resistance could result from a defect in leptin transport to the central nervous system. The likely explanation would be saturation on the soluble form of the leptin receptor, or on the hypothalamic receptor, or either on the response to neuropeptide Y (NPY) [29].

An important mechanism performed by leptin is the inhibition of insulin synthesis and secretion, forming the adipoinsular axis. Hypothalamic leptin signaling regulates hepatic insulin sensitivity via a neurocircuit involving the vagus nerve, contributing to energy balance. The adiposity signals inhibit anabolic circuits and activate catabolic circuits [34].

Leptin also acts on lipid oxidation in skeletal muscle and liver, reducing the synthesis of lipids in the form of triglycerol from monounsaturated fatty acids. It plays important role on the immune, hematopoietic and cardiovascular systems as well. It is a potent modulator of the immune response. The leptin $\mathrm{Ob}-\mathrm{Rb}$ receptor acts on these immunomodulatory products, because it belongs to class I cytokine receptor family, which also includes the receptors for interleukins 2 to 7 , prolactin, and erythropoietin, among others [35].

The leptin is an important modulator of the immune response, especially in innate immunity and its deficit causes susceptibility 
to the deleterious effects of diseases. Leptin promotes the synthesis of proinflammatory cytokines such as TNF- $\alpha$, IL-6, among others, through macrophages-producing cytokines [35].

\section{Obesity and inflammation}

The genesis of obesity is multifactorial, complex and not completely understood. These factors include genetic, environmental and psychological conditions. Abdominal obesity (central obesity) is strongly related to Non-Alcoholic Fatty Liver Disease (NAFLD) and Insulin Resistance (IR), mainly because of the excessive body fat in the central region $[5,17,25]$. These conditions lead to a series of complications due to systemic arterial hypertension, dyslipidemia, and inflammation, which are present in metabolic syndrome (MS). The synergetic effect of these diseases and the accumulation of adipose tissue result in pathological and inflammatory metabolic processes that worsen a bad prognosis [2, 3, 4, 35].

The first researcher to observe that the distribution of body fat could be related to the predisposition to metabolic diseases was Vague (1956) [36]. Numerous epidemiological studies have demonstrated a close relation between IR and a group of different metabolic disorders in subjects with central obesity (also known as apple-shaped obesity), in which case they accumulate fat especially in the intra-abdominal region and the upper region of the thorax [37].

Insulin Resistance (IR) has often been associated to NAFLD, and this plays an important role in the pathophysiology of this illness [21,38-40]. On the other hand, individuals that accumulate more peripheral fat (also known as gynoid or pear-shaped obesity), in which lipid deposits are located mostly in the femoral and gluteal regions, causing fat to accumulate in the subcutaneous tissue, have fewer metabolic complications due to obesity, and are even protected from developing IR and dyslipidemia [37, 39] Heated debates and controversies have emerged in the literature on the mechanisms by which visceral fat becomes more harmful to the body than peripheral fat.

However, obesity is a disorder of energy balance. Both sides of the energy equation, ingestion (intake) and expenditure are regulated by neural and hormonal mechanisms, and so body weight remains constant for many years. This stability is maintained only because of an internal adjustment, capable of identifying the amount of stored energy (adipose tissue) and appropriately regulating food intake and energy expenditure [41, 4].

The neurohumoral mechanisms that regulate energy balance and body weight are very complex. The afferent mechanism generates signals from various locations, and its main components are leptin (adipose tissue), insulin (pancreas), ghrelin (stomach) and peptide YY (ileum and colon) [7]. Leptin decreases food intake. The secretion of ghrelin stimulates appetite and may function as a signal for the beginning of the meal. The peptide YY, which is postprandially released by endocrine cells of the ileum and the colon, is a signal for satiety [33].
Therefore, the adipose tissue account to important metabolic processes, so its imbalance leads to unregulated metabolic functions. This tissue is considered an organ capable of interacting with other organs and systems, mediating obesity related cardiovascular and metabolic complications. In this context, the fat tissue produces an relevant effector, lipoprotein lipase (LPL), which plays a role in inflammatory molecular modulation [42].

Tumor necrosis factor (TNF- $\alpha$ ), produced by adipose tissue macrophages, suppresses the activity conducted by LPL, increasing lipolysis and releasing free fatty acids (FFAs), as a product, into the bloodstream. The endoplasmic reticulum (ER) and FFAs activate the production of TNF- $\alpha$, which is coupled to its specific receptors, producing its biological effects such as apoptosis induction through the activation of nuclear factor $\mathrm{kB}$ (NFkB), the most relevant inflammation effector [43].

The action of LPL is inhibited by TNF- $\alpha$. This cytokine is increased in the presence of adipose tissue accumulation. The hydrolysis of triglycerides and low density lipoprotein (VLDL-C) is conducted by LPL in subjects with central obesity, enhancing inflammation derived from lipidic imbalance [44].

\section{Free fatty acids and the hepatocytes}

Abdominal fat accumulation surrounds the viscera (omentum and mesentery). The liver, which is directly related to the venous drainage of the omentum and the mesentery, through the portal vein system, becomes directly exposed to cytokines and free fatty acids (FFAs) released by visceral adipose tissue. Metabolites from other regions, continuously released by adipose tissue, reach the liver through the portal vein circulation, causing an abundant release of FFAs and proinflammatory cytokines of the visceral adipose tissue (omentum and mesentery). They reach this organ, which stimulates the progression to hepatic insulin resistance and hepatic steatosis in obese individuals [35, 42-44]. The relevance of portal drainage in visceral fat is revealed in several studies about the pathophysiology of NAFLD [45, 39, 46, 47]. These FFAs derived from adipose tissue are factors that intensify the progression of pathological conditions, since the composition of FFAs can be different in obese and thin individuals, which contributes to the damaging effects on liver tissue [48]. Direct contact of visceral fat with proinflammatory substances via the portal system contributes to progression of liver IR observed in patients with steatohepatitis $[45,48]$.

The free fatty acids (FFAs) accumulation makes the adipocyte membrane fragile, resulting in rupture and release of proinflammatory effectors, thereby worsing the inflammatory process through the release of adipocytokines, in a positive feedback [49]. At the same time, this effect stimulates macrophage activation, which leads to a cascade of events that enhance the hepatic damage. This cellular injury results in endoplasmic reticulum (ER) metabolic stress, which compromises this organelle's functions, protein and fat processing. The ER stress causes mitochondrial oxidative stress (OS), which produces reactive oxygen species (ROS) [49, 50]. 


\section{Leptin and Non-Alcoholic Fatty Liver Disease}

The liver plays an importantrole in the processing of hormones, drugs and toxins, and also in the synthesis of metabolically active substances involved in the maintenance of the body homeostasis. It stands out for being the main detoxifying organ, a site of endogenous and exogenous compounds conversion, hormone catabolism, and plasma level regulation, responding to hormonal and neural stimuli that regulate glucose concentration. This organ constitutes only $2 \%$ of adult body weight and uses an average of $20 \%$ of the basal energy expenditure [15].

Non-Alcoholic Fatty Liver Disease (NAFLD) is becoming the most common form of chronic liver disease in the world, and in Western and industrialized countries it affects about $30 \%$ of the population $[39,40,47]$.

NAFLD is classified in three phenotypical group: $i$ ) fatty liver, which is characterized by the presence of an increased liver due to a higher amount of triglycerides in hepatocytes; ii) nonalcoholic fatty liver, when alcohol in not the etiological cause of steatosis; and iii) steatohepatitis, which presents severe lipid accumulation, as well as liver inflammation, and can be caused by alcohol abuse [51].

Steatosis is an etiological condition that refers to any triglyceride accumulation within the parenchyma cells $[15,22$, 52 , 53]. It is mostly observed in the liver because this is the largest organ involved in fat metabolism. Obesity can overload the liver and is one of the main causes of fatty liver. Macroscopic steatosis happens when the amount of fat in the liver exceeds $10 \%$ of liver weight and it is classified as mild if asymptomatic and, histopathologically, until to $30 \%$ of lipid droplets in liver cells [54]. The risk of liver damage is proportional to the amount of fat deposited in hepatocytes. Long-term excessive fat causes damage to hepatocytes throughout several active metabolic pathways, and may maintain chronical inflammation at liver site $[4,10,26]$.

The lipidic stimulus activates the stellate cells (macrophages and Kupffer cells) which produce growth factors, cytokines and chemokines, causing more proliferation and collagen synthesis, in a positive feedback $[34,3,4]$.

Also, the free fatty acids from vicinal adipose tissues requires the bind to apoproteins to form lipoprotein complexes, in which with synthetized triglycerides, are able to enter the circulation. Excessive accumulation of triglycerides can result in defects at any stage, between the entry of fatty acids and the output of the lipoprotein, and is responsible for the occurrence of fatty liver, after several liver injuries $[55,19]$.

\section{Leptin and hepatic steatosis}

Hepatic steatosis is a reversible disease, whose underlying causes can be treated and inhibited in their early stages [24], and the main characteristic of this condition is triglyceride accumulation in hepatocytes, which present no appreciable liver inflammation, neither death of hepatocytes or scarring, although with persistent elevated liver enzymes. Without proper monitoring, fatty liver can progress to chronic liver inflammation, which may progress to liver fibrosis and cirrhosis causing serious complications, including liver failure and hepatocellular carcinoma $[27,52]$.

Two sequential events act as important mechanisms in the establishment and progression of hepatic steatosis: $i$ ) continual fatty acids accumulation in hepatocytes and ii) increase of hepatic oxidative stress [56]. The action of oxidative stress is due to the overload of accumulated liver lipids, resulting in oxidation of these compounds of fatty acids and release of lipid peroxides that produce reactive oxygen species (ROS) [12, 25].

The leptin receptor (Ob-R) is similar to class I cytokine receptors and signal transduction components of IL-6 family of cytokines [53]. The biological actions of leptin will be effective when high amounts of the IL- 6 receptor activate two signaling pathways: i) Janus Kinase (JAK) signal transducers and its activator of transcription, STAT16 and ii) phosphatidylinositol 3-kinase (PI3K); both promoting cell growth, migration and invasion $[23,57]$. Leptin induces the production of inflammatory cytokines (TNF- $\alpha$ and IL-6) that are mostly produced by Kupffer cells of the liver; when they are activated, they intensify local inflammatory responses, resulting in a compensation hepatocyte proliferation (injury proliferation), especially in obese individuals, thereby facilitating the malignant transformation of affected hepatocytes [58].

\section{Leptin, cirrhosis and hepatocellular carcinoma (HCC)}

Leptin, a peptide hormone produced by adipocytes, acts on the energy balance of reproduction and immunomodulation, and this action is linked to the pathogenesis of many diseases [33, $10,26,30,59,60]$. Some authors have been discussing about leptin presenting profibrogenic activity in the liver [25, 61, 62]. High levels of this hormone increase hepatic response to various stimuli of liver fibrosis. Leptin acts to mediate hepatic stellate cell activation and liver fibrosis throughout indirect effects on Kupffer cells. These effects are partially mediated by TGF- $\beta$ (transforming growth factor- $\beta$ ), a significant effector on cirrhosis development and progression [15, 25].

The injury caused by upregulation of TGF- $\beta$ to hepatocytes is triggered by the cascade of inflammatory mediators produced by Kupffer and inflammatory cells, which converge to increase genic expression of TGF- $\beta$. This process mobilizes stellate cells and collagen production $[25,63]$. As result, collagen accumulates gradually, causing a breakdown in the cytoarchitecture, which compromises cellular function, occasioning an increasing cirrhotic phenotype. Wang and collaborators (2010) inferred that leptin may be an important mediator in liver fibrosis, in which Kupffer cells act as facilitators, up regulating TGF- $\beta[20,62,63]$.

The path from steatohepatitis to hepatocellular carcinoma (HCC) has been increasingly documented in the literature [11] and is not a novelty. Leptin acts as a progressor when factors such as age and presence of liver fibrosis are observed $[25,46$, 
$56,61]$. Those factors are significant for the development of HCC in patients with steatohepatitis and it is relevant medicinebased evidence regarding clinical conduct of patients with steatohepatitis and fibrosis, in the early detection diagnosis of HCC.

Lifestyle based on high-fat diets associated with sedentary behavior, smoking, and stress, regardless of socioeconomic status, has played an important role in the obesity epidemic, and its relevance in the public health field is unquestionable [64]. Obesity and type 2 diabetes seem to be risk factors for the development and progression of HCC $[2-4,20,21]$. Since they are closely related to metabolic syndrome, they are also risk factors for NAFLD, reinforcing the importance of the relation between these metabolic conditions and hepatocellular carcinoma [53, 3947].

Although hepatitis is considered the main primary etiology and the most relevant risk factor for development of HCC, the incidence of obesity in adults and children over the past three decades has increased dramatically, which draws the attention of authorities, because, aside represents a public health problem itself, also stands out as an independent risk factor for some malignancies, such as HCC.

\section{Conclusion}

Based on the data presented in this work, it can be concluded that steatosis is common in obese patients, especially in those with visceral obesity. Patients with steatosis mediated by visceral obesity and insulin resistance are more likely to progress to cirrhosis and hepatocellular carcinoma. Although the pathophysiological mechanisms involved in the action of leptin deriving to hepatocellular carcinoma (HCC) are not yet fully described, the literature inferred that leptin is associated with carcinogenesis, especially to HCC.

The increase knowledge regard to non-viral causes of hepatocellular carcinoma can help elucidate to researchers and multidisciplinary professionals about the modifiable risk factors of HCC raise and development. Elegant studies have demonstrated that leptin may induce fibrogenic effects and the evidence of a direct relation between leptin levels and the development of hepatocellular carcinoma, has been frequently reported.

A translational approach to these molecular pathways could enlighten the complexity machinery involved on this important tripod of lectin modulation, liver dysfunction due to lipidic imbalance and the progress of hepatocellular carcinoma.

\section{Acknowledgements}

The authors thank Dr. Bernardo Petriz de Assis for their valuable comments and feedback. This work was possible by the grant UNBDOC 22335/16.

Financial disclosure statement: This work was fund by CNPq, UDF and UnB academic funds.

Conflict-of-interest statement: The authors declare that there are no conflicts of interest.

\section{References}

1. Aleksandrova K, Boeing H, Nöthlings U, Jenab M, Fedirko V, Kaaks R, et al. Inflammatory and metabolic biomarkers and risk of liver and bilary tract cancer. Hepatology. 2014;60(3):858-871. doi: 10.1002/ hep.27016.

2. Caldwell SH, Crespo DM, Kang HS, Al-Osaimi AMS. Obesity and hepatocellular carcinoma. Gastroenterology. 2004;127(5, Supplement 1): S97-S103.

3. Toffanin S, Friedman SL, Llovet JM. Obesity, Inflammatory Signaling, and Hepatocellular Carcinoma-An Enlarging Link. Cancer Cell. 2010;17(2):115-117. doi: 10.1016/j.ccr.2010.01.018.

4. El-Serag HB \& Kanwa F. Obesity and hepatocellular carcinoma: Hype and reality. Hepatology. 2014;60(3):779-781. doi: 10.1002/ hep. 27172 .

5. Pocha C, Kolly P, Dufour JF. Nonalcoholic Fatty Liver Disease-Related Hepatocellular Carcinoma: A Problem of Growing Magnitude. Seminars in Liver Disease. 2015; 35(3):304-317. doi: 10.1055/s0035-1562949.

6. Reeves HL, Zaki MYW, Day CP. Hepatocellular Carcinoma in Obesity, Type 2 Diabetes, and NAFLD. Digestive Diseases and Sciences. 2016;61(5):1234-1245.

7. Stefanou N, Papanikolaou V, Furukawa Y. Leptin as a critical regulator of hepatocelular carcinoma development through modulation of human telomerase reverse transcriptase. BMC Cancer. 2010;10:442.

8. Huang L and Li C. Leptin: A multifunctional hormone. Celular Res. 2000;10(2):81-92.

9. Fantuzzi G. Adipose tissue, adipokines and inflammation. J Allergy Clin Immunol. 2005;115(5):911-919.

10. Rosenbaum M and Leibel RL. 20 YEARS OF LEPTIN: Role of leptin in energy homeostasis in humans. J Endocrinol. 2014;223(1):T83-T96. doi: 10.1530/JOE-14-03.

11.Wang SN, Lee KT, Ker CG. Leptin in hepatocellular carcinoma. World J Gastroenterology. 2010;16(46):5801-5809.

12. Martins, MC, Lima FL, Fonseca A. Relação entre a leptina, massa corporal e a síndrome metabólica numa amostra da população adulta. Revista Portuguesa de cardiologia. 2012;31(11): 711-719.

13. Caro JF, Kolaczynski JW, Nyce MR, Ohannesian JP, Opentanova I, Goldman WH, et al. Decreased cerebrospinal-fluid/serum leptin ratio in obesity: a possible mechanism for leptin resistance. Lancet. 1996;348(9021):159-161.

14. Parkin DM, Bray F, Ferlay J, Pisani P. Global cancer statistics, 2002. CA Cancer J Clin, 2005;55(2):74-108.

15. Tarantino G, Finelli C. Pathogenesis of hepatic steatosis: The link between hypercortisolism and non-alcoholic fatty liver disease. World J Gastroenterol. 2013;19(40):6735-6743. doi: 10.3748/wjg. v19.i40.6735.

16. El-Serag HB. Epidemiology of Viral Hepatitis and Hepatocellular Carcinoma. Gastroenterology. 2012;142(6):1264-1273. doi: 10.1053/j.gastro.2011.12.061.

17. Dongiovanni P, Romeo S, Valenti L. Hepatocellular carcinoma in nonalcoholic fatty liver: Role of environmental and genetic factors. World J Gastroenterol. 2014;20(36): 12945-12955. doi: 10.3748/wjg. 


\section{v20.i36.12945}

18. Llovet Josep M, Zucman-Rossi J, Pikarsky E, Sangro B, Schwartz M, Sherman M, et al . Hepatocellular carcinoma. Nature Reviews Disease Primers. 2016;2:16018. doi: 10.1038/nrdp.2016.18.

19. Catalán V, Gómez-Ambrosi J, Rodríguez A, Ramírez B, Valentí V, Moncada R, et al. Peripheral mononuclear blood cells contribute to the obesity-associated inflammatory state independently of glycemic status: involvement of the novel proinflammatory adipokines chemerin, chitinase-3-like protein 1, lipocalin-2 and osteopontin. Genes \& Nutrition. 2015;10(3):460. doi:10.1007/s12263-015-0460

20. Rodríguez-Hernández H, Simental-Mendía LE, Rodríguez-Ramírez G, Reyes-Romero MA. Obesity and Inflammation: Epidemiology, Risk Factors, and Markers of Inflammation. International Journal of Endocrinology. 2013. doi:10.1155/2013/678159.

21. Malik VS, Willett WC, Hu FB. Global obesity: trends, risk factors and policy implications. Nature Reviews Endocrinology 2013;9(1):13-27. doi:10.1038/nrendo.2012.199.

22. Wree A, Kahraman A, Gerken G, Canbay A. Obesity Affects the Liver The Link between Adipocytes and Hepatocytes. Digestion, 2011;83(12):124-133. doi:10.1159/000318741.

23. Wang SN, Lee KT, Ker Chen-G. Leptin in hepatocellular carcinoma World J Gastroenterology. 2010;16(46):5801-5809.

24.Sten E, Karl F. Nonalcoholic Steatohepatitis in Obesity: A Reversible Condition. Journal of internal medicine. 1986; 220(1):83-88.

25. Magkos F, Fabbrini E, Klein S. Pathogenesis of Hepatic Steatosis and Fibrosis: Role of Leptin. Leptin. 2015:89-101. Leptin Regulation and Clinical Applications. Editors: Sam Dagogo-Jack.

26. Polyzosa SA, Kountourasa J, Mantzoros CS. Leptin in nonalcoholic fatty liver disease: A narrative review. Metabolism. 2015;64(1):6078. doi:10.1016/j.metabol.2014.10.012

27. Park EJ, Lee JH, Yu GY, He G, Ali SR, Holzer RG, et al. Dietary and Genetic Obesity Promote Liver Inflammation and Tumorigenesis by Enhancing IL-6 and TNF Expression. Cell. 2010;140(2):197-208. doi:10.1016/j.cell.2009.12.052.

28. Zhang Y, Proença R, Maffei M, Barone M, Leopold L, Friedman JM. Positional cloning of the mouse obese gene and its human homologue. Nature. 1994;372(6505):425-432. DOI:10.1038/372425a0.

29. Rodrigues AM, Radominski RB, Suplicy HL, Almeida SM, Niclewicz PA, Boguszewski CL. The cerebrospinal fluid/serum leptin ratio during pharmacological therapy for obesity. J Clin Endocrinol Metab 2002;87(4):1621-1626. DOI: 10.1210/jcem.87.4.8420.

30. Li Z, Ceccarini G, Eisenstein M, Tan K, Friedman JM. Phenotypic effects of an induced mutation of the ObRa isoformof the leptin receptor. Molecular Metabolism 2. 2013;2(4):364-375. doi:10.1016/j molmet.2013.07.007.

31. Hoggard N, Hunter L, Duncan JS, Williams LM, Trayhurn P, Mercer JG. Leptin and leptin receptor mRNA and protein expression in the murine fetus and placenta. Proc. Natl. Acad. Sci. 1997;94:11073-11078.

32.Huang L \& Wang Z. Modulation of circulating leptin levels by its soluble receptor. J BiolChem. 2001;276(9):6343-6349. DOI:10.1210/ jcem.87.4.8420.

33. Klok MD, Jakobsdottir S, Drent ML. The role of leptin and ghrelin in the regulation of food intake and body weight in humans: a review.
Obes Rev. 2007;8(1):21-34. DOI:10.1111/j.1467-789X.2006.00270.x.

34. Wellen KE \& Hotamisligil GS. Inflammation, stress, and diabetes. J Clin Invest. 2005;115:1111-1119. DOI:10.1172/JCI25102.

35. Fruhhbeck G, Gomez-Ambrosi J, Muruzabal FJ, Burrell MA. The adipocyte: a model for integration of endrocrine and metabolic signaling in energy metabolism regulation. Am J Physiol Endocrinol Metab. 2001;280:E827-E847.

36. Vague J. The degree of masculine differentiation of obesities: a factor determining predisposition to diabetes, atherosclerosis, gout, and uric calculous disease. Am J Clin Nutr. 1956;4(1):20-34

37. Tanko LB, Bagger YZ, Alexandersen P, Larsen PJ, Christiansen C. Peripheraladiposity exhibits an independentdominantantiatherogenic effect in elderly women. Circulation. 2003;107(12):1626-1631. DOI:10.1161/01.CIR.0000057974.74060.68.

38. Marchesini G, Brizi M, Morselli-Labate AM, Bianchi G, Bugianesi E, McCullough AJ, et al. Association of nonalcoholic fatty liver disease with insulin resistance. Am J Med. 1999;107(5):450-455

39. Gaggini M, Morelli M, Buzzigoli E, DeFronzo RA, Bugianesi E, Gastaldelli A. Non-Alcoholic Fatty Liver Disease (NAFLD) and Its Connection with Insulin Resistance, Dyslipidemia, Atherosclerosis and Coronary Heart Disease. Nutrients. 2013;5(5):1544-1560. doi:10.3390/nu5051544.

40. Privitera G, Spadaro L, Corradina Alagona,, Salvatore Calanna, Salvatore Piro, Agata Maria Rabuazzo, et al. Hepatic insulin resistance in NAFLD: relationship with markers of atherosclerosis and metabolic syndrome components. Acta Diabetologica. 2015: 1-11. Published online: October 2015. doi:10.1007/s00592-015-0816-y.

41. Kershaw EE, Flier JS. Adipose tissue as an endocrine organ. J Clin Endocrinol Metab. 2004;89(6):2548-2556. DOI:10.1210/jc.20040395.

42. Berg AH \& Scherer PE. Adipose tissue, inflammation, and cardiovascular disease. Circ Res. 2005;96(9):939-949. Doi:10.116101. RES.0000163635.62927.34.

43. Gregor MF \& Hotamisligil GS. Thematic review series: Adipocyte Biology. Adipocyte stress: the endoplasmic reticulum and metabolic disease. J Lipid Res. 2007;48(9):1905-1914. DOI:10.1194/jlr. R700007-JLR200.

44. Miyazaki Y, Pipek R, Mandarino LJ, DeFronzo RA. Tumor necrosis factor alpha and insulin resistance in obese type 2 diabetic patients. Int J ObesRelat Metab Disord. 2003;27(1):88-94. DOI:10.1038/ sj.ijo.0802187.

45. Catalano KJ, Stefanovski D, Bergman RN. Critical role of the mesenteric depot versus other intra-abdominal adipose depots in the development of insulin resistance in young rats. Diabetes. 2010;59(6):1416-1423. doi:10.2337/db08-0675.

46. Ebbert JO \& Jensen MD. Fat Depots, Free Fatty Acids, and Dyslipidemia Nutrients 2013;5(2):498-508. doi:10.3390/nu5020498.

47. Leamy AK, Egnatchik RA, Young JD. Molecular mechanisms and the role of saturated fatty acids in the progression of non-alcoholic fatty liver disease. Progress in Lipid Research. 2013;52(1):165-174. doi:10.1016/j.plipres.2012.10.004

48. Miles JM, Jensen MD. Counterpoint: visceral adiposity is not causally related to insulin resistance. Diabetes Care. 2005;28(9):2326-2328. Doi:10.2337diacare.28.9.2326.

49. Hosogai N, Fukuhara A, Oshima K, Miyata Y, Tanaka S, Segawa K, et 
al. Adipose tissue hypoxia in obesity and its impact on adipocytokine dysregulation. Diabetes. 2007;56(4):901-911. DOI:10.1038/ sj.ijo.0802187.

50. Ozcan U, Cao Q, Yilmaz E, Lee AH, Iwakoshi NN, Ozdelen E, et al Endoplasmic reticulum stress links obesity, insulin action, and type 2 diabetes. Science. 2004;306(5695):457-461. DOI:10.1126/ science. 1103160

51. de Alwis NM, Day CP. Non-alcoholic fatty liver disease: the mist gradually clears. J Hepatol. 2008;48(Suppl 1):S104S112. doi:10.1016/j.jhep.2008.01.009.

52. Starley QB, Calcagno JC. Nonalcoholic fatty liver disease and hepatocellular carcinoma: weighty connection. Hepatology. 2010;51(5):1820-1832.

53. Sun B, Karin M. Obesity, inflammation, and liver cancer. Journal of Hepatology. 2012;56(3):704-713. doi:10.1016/j.jhep.2011.09.020.

54. Martins AMA, Coelho GR, Marques GA, Moraes MO, Valença-Jr JT Huygens JPG. Hepatic Steatosis Assessment: A comparative study between surgeon evaluation and forward histopathologic findings. Arq Gastroenterol. 2013;50(1):15-18.

55. Day CP \& James OFW. Steatohepatitis: a tale of two "hits"? Gastroenterology. 1998;114(4):842-845. DOI:10.1016/S00165085(98)70599-2.

56. Tariq Z, Green CJ, Hodson L. Are oxidative stress mechanisms the common denominator in the progression from hepatic steatosis towards non-alcoholic steatohepatitis (NASH)? Liver International. 2014;34(7):e180-e190. DOI:10.1111/liv.12523.

57. Nakagawa H \& Maeda S. Inflammation and stress-related signaling pathways in hepatocarcinogenesis. World J Gastroenterology. 2012;18(31):4071-4081. doi:10.3748/wjg.v18. i31.4071.

58. Dutta D, Ghosh S, Pandit K. Leptin and cancer: pathogenesis and modulation. Indian Journal Endocrinol Metab. 2012; 16(Suppl 3):S596-S600. doi:10.4103/2230-8210.105577.

59. Blüher M \& Mantzoros CS. From leptin to other adipokines in health and disease: Facts and expectations at the beginning of the 21st century. Metabolism. 2015;64(1):131-145. doi: 10.1016/j. metabol.2014.10.016.

60. Procaccinia C, Pucinob V, Mantzorosc CS, Matarese G. Leptin in autoimmune diseases. Metabolism. 2015;64(1):92104. doi:10.1016/j.metabol.2014.10.014.

61. Coombesa JD, Choic SS, Swiderska-Sync M, Mankaa P, Reidf DT, Palmab E, et al. Osteopontin is a proximal effector of leptin-mediated nonalcoholic steatohepatitis (NASH) fibrosis. Biochimica et Biophysica Acta (BBA) - Molecular Basis of Disease. 2016;1862(1):135-144. doi:10.1016/j.bbadis.2015.10.028.

62.Abu-Taira L, Dorona S, Mahamidb M, Amera J, Safadia R. Leptin modulates lymphocytes' adherence to hepatic stellate cells is associated with oxidative status alterations. Mitochondrion. 2013;13(5):473-480. doi:10.1016/j.mito.2012.10.012.

63. Parka Ji-H, Yoona J, Leeb KYong, Park B. Effects of geniposide on hepatocytes undergoing epithelial-mesenchymal transition in hepatic fibrosis by targeting TGFß/Smad and ERK-MAPK signaling pathways. Biochimie. 2015;113:26-34. doi:10.1016/j.biochi.2015.03.015.

64. Mancini MC \& Halpern A. Obesidade: como diagnosticar e tratar. Rev Med Bras. 2006;63:132-143. 